\title{
Experimentally verified multi-objective iterative learning control design with frequency domain specifications
}

\author{
Wojciech Paszke, Krzysztof Patan, Eric Rogers
}

\begin{abstract}
This paper considers the design of multi-objective iterative learning control (ILC) schemes for discrete linear systems. In particular, a two step design approach is developed where the feedback and learning controllers are designed separately. The design procedures developed are able to impose performance specifications over selected frequency ranges to obtain the desired shapes of the sensitivity functions relevant to a particular example. This makes it possible to reject disturbances in specific frequency ranges and hence the desired performance specifications for both the transient response and error convergence speed can be achieved. All design computations required can be completed using linear matrix inequalities (LMIs). Preliminary experimental results demonstrate the effectiveness of the new design.
\end{abstract}

\section{INTRODUCTION}

In many applications, e.g. pick and place robots and batch chemical processes, the system has to follow the same trajectory (reference signal) repeatedly. If feedback control is only applied and the reference signal is a dominant disturbance, the tracking error is (approximately) the same for each repetition of a given task. Iterative Learning Control (ILC) can be introduced into the control system to reduce the repetitive part of the tracking error during subsequent repetitions with a feedforwad signal update. Specifically, ILC is a feedforward control scheme for improving the tracking response of systems that repeat a given task or operation defined over a finite duration. Each repetition is known as a trial, or pass, and when a trial is complete, the system resets to the same initial conditions and the next trial can begin, either immediately or after a further period of time has elapsed.

The core advantage of this control structure is the use information from the previous trial to update the control input applied on the next trial and thereby improve the trial-to-trial performance. This feature is a major reason why ILC has been extensively employed in high precision control systems and in other area, see, [1], [2] as the fundamental works in these aspects.

In application, the objective of ILC is to construct the control input signal such that the output tracks the reference as accurately as possible. Let $\left\{e_{k}\right\}_{k}$ denote the error sequence generated over the trials. Then the basic ILC problem

W. Paszke and K. Patan are with the Institute of Control and Computation Engineering,University of Zielona Góra, ul. Szafrana 2, 65-516 Zielona Góra, Poland. E-mail: \{w.paszke, k.patan\}@issi.uz.zgora.pl

E. Rogers is with the Department of Electronics and Computer Science, University of Southampton, Southampton SO17 1BJ, UK. Email: etareecs.soton.ac.uk

This work is partially supported by National Science Centre in Poland, grant No. 2014/15/B/ST7/03208 is to design control action to ensure that $\left\{e_{k}\right\}_{k}$ converges in $k$. Also once a trial is complete in ILC, all information generated is available for use in design. One of the popular ILC laws that includes previous trial information is

$$
u_{k+1}(p)=u_{k}(p)+L e_{k}(p),
$$

where $u_{k}(p)$ denotes the input on trial $k$ and time instant $p, L$ acts on the error at the previous trial and it is also often termed the learning filter in the literature. Frequently, the ILC law is augmented with a feedback controller that stabilizes the system and suppresses unknown disturbances. This construction results in a so-called current trial ILC scheme. The learning controller is designed to guarantee convergence in the trial domain and in many cases its construction is based on the inverse of the plant dynamics (see the relevant references in [2]) resulting from the design and application of the feedback loop.

The design of the feedback and learning controllers must include some requirements on transient dynamics and trialto-trial error convergence. However, these requirements are often defined over the complete frequency spectrum. This is a very strict condition since design requirements and specifications are mostly defined for different frequency ranges of relevance. For example, a closed-loop feedback control system should have small sensitivity in a low frequency range and small complementary sensitivity in the high frequency range. Furthermore, since the bandwidth of the reference signal has the strongest influence on the convergence rate, learning over this frequency range should only occur when the ILC scheme is applied.

The contribution of this paper is to provide new insights into the currently known two step ILC design procedures. Specifically, systematic guidelines for designing of ILC schemes over limited frequency range are proposed. The generalized version of Kalman-YakubovichPopov (KYP) lemma [3] is extensively used to permit controller design over selected frequency ranges. These controller design procedures can also include multiple design specifications (e.g., reject disturbances at specific frequencies), whereas the vast majority of currently known designs cannot impose many relevant additional performance specifications. In particular, the developed results allows a designer to specify and/or maximize, frequency ranges where the error convergence condition have to be satisfied. Furthermore, the design is based on using controller parametrization in a finite impulse response (FIR) filter form. This allows controller design procedures over convex sets and therefore they are amenable to effective algorithmic solution in terms of LMIs. 
The notation adopted in this paper is as follows. The null and identity matrices with compatible dimensions are denoted by 0 and $I$ respectively. The notation $X \succ Y$ (respectively $X \prec Y$ ) means that the symmetric matrix $X-Y$ is positive definite (respectively negative definite). The symbol $(\star)$ denotes block entries in symmetric matrices and $\rho(\cdot)$ and $\bar{\sigma}(\cdot)$ denote the spectral radius and maximum singular value of their matrix arguments, respectively. Finally, the superscript $*$ denotes the complex conjugate transpose of a matrix and $\otimes$ the matrix Kronecker product.

The analysis in the remainder of this paper makes use of the following result, known as the generalized KYP lemma.

Lemma 1: [3] For a given linear discrete time-invariant system with the transfer-function matrix $M(z)$ and the frequency response matrix $M\left(e^{j \theta}\right)=C\left(e^{j \theta} I-A\right)^{-1} B+D$, the following inequalities are equivalent

(i) $\left[\begin{array}{c}M\left(e^{j \theta}\right) \\ I\end{array}\right]^{*} \Pi\left[\begin{array}{c}M\left(e^{j \theta}\right) \\ I\end{array}\right] \prec 0, \quad \forall \theta \in \Theta$,

where $\Pi$ is a given real symmetric matrix and $\Theta$ denotes the frequency ranges (see Table I).

(ii)

$$
\left[\begin{array}{cc}
A & B \\
I & 0
\end{array}\right]^{T}(\Phi \otimes P+\Psi \otimes R)\left[\begin{array}{cc}
A & B \\
I & 0
\end{array}\right]+\left[\begin{array}{cc}
C & D \\
0 & I
\end{array}\right]^{T} \Pi\left[\begin{array}{cc}
C & D \\
0 & I
\end{array}\right] \prec 0,
$$

where $R \succ 0, P$ is a symmetric matrix, $\Phi=$ $\operatorname{diag}\{-1,1\}$ and $\Psi$ is specified with the reference to the chosen frequency range - see the below Table I.

TABLE I

FREQUENCY RANGES OF INTEREST

\begin{tabular}{|c|c|c|c|}
\hline & LF (low freq.) & MF (middle freq.) & HF (high freq.) \\
\hline$\Theta$ & $|\theta| \leq \varpi_{l}$ & $\varpi_{1} \leq \theta \leq \varpi_{2}$ & $|\theta| \geq \varpi_{h}$ \\
\hline$\Psi$ & {$\left[\begin{array}{cc}0 \\
1-2 \cos \left(\varpi_{l}\right)\end{array}\right]$} & $\left.\begin{array}{cc}0 & e^{j \varpi_{a}} \\
e^{-j \varpi_{a}}-2 \cos \left(\varpi_{b}\right)\end{array}\right]$ & {$\left[\begin{array}{c}0 \\
-1 \\
-12 \cos \left(\varpi_{h}\right)\end{array}\right]$} \\
\hline
\end{tabular}

where $\varpi_{a}=\frac{\varpi_{1}+\varpi_{2}}{2}$ and $\varpi_{b}=\frac{\varpi_{2}-\varpi_{1}}{2}$.

\section{PRoblem Formulation}

This paper addresses the case when the plant dynamics to be controlled can be modeled by the following discrete linear time-invariant state-space model over $0 \leq p \leq \alpha-1, k \geq 0$ as written in the ILC setting as

$$
\begin{aligned}
x_{k}(p+1) & =A x_{k}(p)+B u_{k}(p), \\
y_{k}(p) & =C x_{k}(p),
\end{aligned}
$$

where $\alpha<\infty$ denotes the finite trial length, $x_{k}(p) \in \mathbb{R}^{n}$, $y_{k}(p) \in \mathbb{R}^{m}$ and $u_{k}(p) \in \mathbb{R}^{l}$ are the state, output and input vectors at the $k$-th trial respectively.

The block diagram of the considered ILC is shown in Fig. 1. It consists of a unity negative feedback control loop with the feedback controller $C(z)$ applied on the current trial $k$ to ensure stability and/or the required along the trial dynamics. The memory block stores the previous trial information which is used for the computation of the current trial control and $Y_{d}(z)$ denotes the reference. Commonly, $L(z)$ stands for the learning controller or filter and $Q(z)$ is the robustness filter. All computations carried out in the

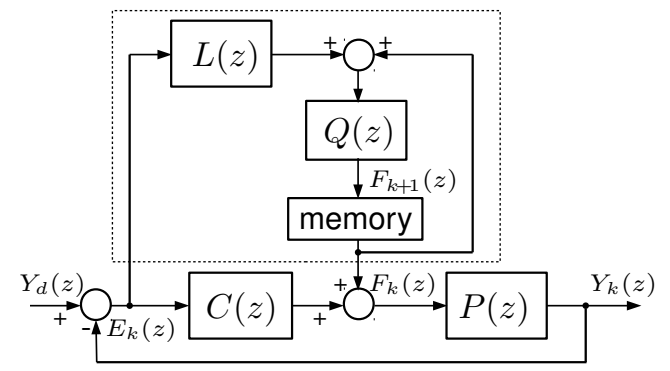

Fig. 1. ILC block diagram representation.

framework of the dashed box are completed off-line during the time elapsed between the end of one trial and the beginning of another one. According to Fig. 1 the ILC law can be represented as follows:

$$
F_{k+1}(z)=Q(z)\left(F_{k}(z)+L(z) E_{k}(z)\right),
$$

and hence the previous trial error feedforward contribution (assuming $Y_{d}(z)=0$ ) to the current trial error is

$$
E_{k}(z)=-\left[(I+P(z) C(z))^{-1} P(z)\right] F_{k}(z)=-S_{P}(z) F_{k}(z),
$$

where $S_{P}(z)=(I+P(z) C(z))^{-1} P(z)$ denotes the process sensitivity function and the propagation of the error from trial-to-trial is given by

$$
E_{k+1}(z)=Q(z)\left(I-S_{P}(z) L(z)\right) E_{k}(z) .
$$

Introducing

$$
M(z)=Q(z)\left(I-S_{P}(z) L(z)\right),
$$

it follows that the condition for trial-to-trial error convergence can been formulated in $\mathcal{H}_{\infty}$ control terms as [2]:

$$
\|M(z)\|_{\infty} \triangleq \sup _{\theta \in[-\pi, \pi]} \bar{\sigma}\left(M\left(\mathrm{e}^{\mathrm{j} \theta}\right)\right)<1,
$$

and minimizing $\|M(z)\|_{\infty}$ increases the trial-to-trial convergence speed.

Clearly, based on (3) and (4) we have that fast error convergence will occur if $L(z) \approx S_{P}^{-1}(z)$ for the entire frequency range. However, if $S_{P}(z)$ is strictly proper its exact inverse is improper. Hence, we will limit our attention to the frequency range where $L(z)$ can be a good approximation to $S_{P}^{-1}(z)$. Undesirable frequencies should be cut-off by the $Q(z)$ because the inverse of $S_{P}(z)$ is not sufficiently matched at higher frequencies. The next sections consider the separate design of $C(z)$ and $L(z)$ to satisfy the error convergence condition in finite frequency range only and then a suitable cut-off frequency of $Q(z)$ is established.

\section{Parametrization of a Feedback CONTROller}

Having obtained conditions for the ILC convergence, there exist a need for framework to impose specifications beyond the trial-to-trial error convergence only. In particular, it is required to attenuate influence of some exogenous signals (such as disturbances or noises) to meet the control performance specifications. Hence, this section starts with a more 
general model of a plant than (2). Let consider a discretetime system of the following form over $0 \leq p \leq \alpha-1, k \geq 0$,

$$
\begin{aligned}
x_{k}(p+1) & =A x_{k}(p)+B_{x_{1}} w_{k}^{1}(p)+B_{x_{2}} w_{k}^{2}(p)+B u_{k}(p) \\
z_{k}^{1}(p) & =C_{z_{1}} x_{k}(p)+D_{z_{1}} w_{k}^{1}(p)+D_{1} u_{k}(p) \\
z_{k}^{2}(p) & =C_{z_{2}} x_{k}(p)+D_{z_{2}} w_{k}^{2}(p)+D_{2} u_{k}(p) \\
y_{k}(p) & =C x_{k}(p)+D_{y_{1}} w_{k}^{1}(p)+D_{y_{2}} w_{k}^{2}(p)
\end{aligned}
$$

where $w^{i} \in \mathbb{R}^{r}$ is the disturbance input, $z^{i} \in \mathbb{R}^{q_{i}}$ is the controlled output and $y \in \mathbb{R}^{m}$ is the measured output. $A, B$, $B_{x_{i}}, C, C_{z_{i}}, D_{i}, D_{z_{i}}$ and $D_{y_{i}}$ are known system matrices of the suitable dimensions. Also, let a state-space representation of the feedback controller $C(z)$ be given by

$$
\begin{aligned}
x_{k}^{c}(p+1) & =A_{c} x_{k}^{c}(p)+B_{c} y_{k}(p), \\
u_{k}(p) & =C_{c} x^{c}(p)+D_{c} y(p),
\end{aligned}
$$

where $x^{c} \in \mathbb{R}^{n_{c}}$ is the state vector of the controller and $A_{c}$, $B_{c}, C_{c}, D_{c}$ are controller matrices of appropriate dimensions to be designed. Clearly, the state-space model (6) results in the controller realization $C(z)=D_{c}+C_{c}\left(z I-A_{c}\right)^{-1} B_{c}$.

It is known that multi-objective control problems are hard to solve when the controller structure (6) is directly applied. The crucial problem is that the closed-loop transfer function matrix, on which design specifications are imposed, is nonlinearly dependent on the controller $C(z)$. Hence, the resulting design conditions are nonconvex and no computationally effective method exists for checking them. Fortunately, a multi-objective control problem can be reformulated to the form of convex optimization problem by applying a particular set of transformations as proposed in [4]. To proceed, the controller (6) is transformed to the observerbased structure by defining

$$
\hat{x}_{k}(p+1)=\left(A+B D_{c} C-B K-L C\right) \hat{x}_{k}(p)+L y_{k}(p)+B v_{k}(p),
$$

where $\hat{x}_{k}(p)$ in an estimate of the state vector, $v_{k}(p)$ is additional signal to be used later, $L$ is the observer gain and $K$ is the state feedback gain matrix. Thus, the control law takes the form

$$
u_{k}(p)=-K \hat{x}_{k}(p)+v_{k}(p) .
$$

Clearly, the matrices $K$ and $L$ can be computed using approaches as the LQG control (for instance, $K$ and $L$ can be derived using MATLAB routines dlqr and dlqe). Next, let define the state estimation error $e_{k}(p)=y_{k}(p)-C \hat{x}_{k}(p)$. Using the observer (7) and the control law (8), the system (5) along with the controller (6) can be transformed to the closed-loop system represented as follows:

$$
\left[\begin{array}{c}
z_{k}^{i}(p) \\
e_{k}(p)
\end{array}\right]=\left[\begin{array}{c:c}
\mathcal{A}_{i} & \mathcal{B}_{i} \\
\hdashline \mathcal{C}_{i} & \mathcal{D}_{i}
\end{array}\right]\left[\begin{array}{c}
w_{k}^{i}(p) \\
v_{k}(p)
\end{array}\right]
$$

where $i=1,2$ and

$$
\begin{gathered}
\mathcal{A}_{i}=\left[\begin{array}{cc}
A+B D_{c} C-B K & B K \\
0 & A+B D_{c} C-L C
\end{array}\right], \\
\mathcal{B}_{i}=\left[\begin{array}{cc}
B_{x_{i}}+B D_{c} D_{y_{i}} & B \\
B_{x_{i}}+B D_{c} D_{y_{i}}-L D_{y_{i}} & 0
\end{array}\right],
\end{gathered}
$$

$$
\mathcal{C}_{i}=\left[\begin{array}{cc}
C_{z_{i}}+D_{i} D_{c} C-D_{i} K & D_{i} K \\
0 & C
\end{array}\right], \mathcal{D}_{i}=\left[\begin{array}{cc}
D_{z_{i}}+D_{i} D_{c} D_{y_{i}} & D_{i} \\
D_{y_{i}} & 0
\end{array}\right] .
$$

In the operator domain the equation (9) takes the form:

$$
\left[\begin{array}{c}
Z_{k}^{i}(z) \\
E_{k}(z)
\end{array}\right]=\left[\begin{array}{cc}
G_{i 1}(z) & G_{i 2}(z) \\
G_{i 3}(z) & 0
\end{array}\right]\left[\begin{array}{c}
W_{k}^{i}(z) \\
V_{k}(z)
\end{array}\right]
$$

where $G_{i 1}(z)$ stands for the open-loop transfer function matrix from $w_{i}$ to $z_{i}, G_{i 2}(z)$ is the open-loop transfer function matrix from $v$ to $z_{i}$ and $G_{i 3}(z)$ represents the openloop transfer function matrix from $w_{i}$ to $e$. The next step is to develop an effective method for computing the feedback controller matrices (6) with performance specification. This can be achieved by means of Youla parametrization. To proceed, assume the signal $v$ in (8) be as follows

$$
v_{k}(p)=H e_{k}(p)
$$

where $H$ represents the Youla parameter. The Youla parameter is used to form a closed-loop transfer function which represent mapping from $w^{i}$ to $z^{i}$. Specifically, for each channel we have

$$
G_{i}(z)=G_{i 1}(z)+G_{i 2}(z) H(z) G_{i 3}(z)
$$

and Youla parameter $H(z)$ is selected in the form of a FIR system

$$
H(z)=h_{0}+h_{1} z^{-1}+\cdots+h_{n} z^{-n}
$$

Then, the state-space representation of $H(z)$ is

$$
H(z)=\left[\begin{array}{c:c}
A_{h} & B_{h} \\
\hdashline C_{h} & D_{h}
\end{array}\right]=\left[\begin{array}{ccccc:c}
0 & 1 & 0 & \cdots & 0 & 0 \\
0 & 0 & 1 & \cdots & 0 & 0 \\
\vdots & \vdots & \ddots & \ddots & \vdots & \vdots \\
0 & 0 & 0 & \cdots & 1 & 0 \\
0 & 0 & 0 & \cdots & 0 & 1 \\
\hdashline \overline{h_{n}} & \overline{h_{n-1}} & \cdots & \overline{h_{2}} & h_{1} & \overline{h_{0}}
\end{array}\right],
$$

In view of representations (9) and (10), the state space model matrices representing $G_{i 1}, G_{i 2}$ and $G_{i 3}$ can be easily found. Due to space limitations, the details of these matrices are not provided here but can be found e.g. in [5]. Assuming that these matrices are related to single input single output systems, $G_{i}$ in (11) can be obtained via series connection of $G_{i 2}$ and $G_{i 3}$ first, then the achieved representation is connected in series with $H(z)$ and finally via parallel connection with $G_{i 1}$. Resulting representation of $G_{i}$ in the packed matrix is

$$
G_{i}=\left[\begin{array}{c:c}
\mathbb{A}_{i} & \mathbb{B}_{i} \\
\hdashline \mathbb{C}_{i} & \mathbb{D}_{i}
\end{array}\right]
$$

Due to a complicated form as well as large space required, details concerning matrices $\mathbb{A}_{i}, \mathbb{B}_{i}, \mathbb{C}_{i}, \mathbb{D}_{i}$ are not provided here but can be easily determined via routine matrix transformations. 


\section{A. Design specification in frequency domain}

In order to define frequency requirements for the control system one can use the principal gains approach. Principal gains (singular values) can be used to assess the performance of the system. The control synthesis problem is to design the control system represented by the closed-loop transfer function matrices $G_{i}(z)$ in such a way as to satisfy mixed frequency specifications

$$
\bar{\sigma}\left(G_{i}\left(\mathrm{e}^{\mathrm{j} \theta}\right)\right) \leq \gamma_{i}, \quad \forall \theta \in \Theta_{i},
$$

where $\gamma_{i}$ is the performance bound and $\Theta_{i}$ denotes the frequency ranges of relevance as in Table I. Furthermore, select the matrix $\Pi$ as

$$
\Pi=\left[\begin{array}{cc}
I & 0 \\
0 & -\gamma_{i}^{2} I
\end{array}\right],
$$

where $\gamma_{i}$ is a given scalar that satisfies $0<\gamma_{i} \leq 1$. Then

$$
\bar{\sigma}\left(G_{i}\left(e^{j \theta}\right)\right)<\gamma_{i} \Leftrightarrow\left\|G_{i}\left(e^{j \theta}\right)\right\|_{\infty}<\gamma_{i}, \forall \theta \in \Theta_{i} .
$$

Next, to meet finite frequency required specifications, following [3], block entries of the matrix $\Psi$ in (1) depend on the chosen frequency range and are defined in Lemma 1. Then straightforward adaptation of Lemma 1 lead to the following result.

Theorem 1: Let $\Psi=\left[\begin{array}{cc}\Psi_{11} & \Psi_{12} \\ \Psi_{12}^{*} & \Psi_{22}\end{array}\right]$ be given. For the state-space realization of $G_{i}$ in the form (14) if there exist matrices $R_{i} \succ 0$ and symmetric $P_{i}$ such that the following LMIs

$$
\begin{aligned}
& {\left[-\mathbb{A}_{i}^{T} P_{i} \mathbb{A}_{i}+\Psi_{11} \mathbb{A}_{i}^{T} R_{i} \mathbb{A}_{i}+\Psi_{12} \mathbb{A}_{i}^{T} R_{i}+\left(\Psi_{12} \mathbb{A}_{i}^{T} R_{i}\right)^{*}+P_{i}+\Psi_{22} R_{i}\right.} \\
& -\mathbb{B}_{i}^{T} P_{i} \mathbb{A}_{i}+\Psi_{11} \mathbb{B}_{i}^{T} R_{i} \mathbb{A}_{i}+\Psi_{12} \mathbb{B}_{i}^{T} R_{i} \\
& \left.\begin{array}{cc}
-\mathbb{A}_{i}^{T} P_{i} \mathbb{B}_{i}+\Psi_{11} \mathbb{A}_{i}^{T} R_{i} \mathbb{B}_{i}+\Psi_{12}^{*} R_{i} \mathbb{B}_{i} & -\mathbb{C}_{i}^{T} \\
-\mathbb{B}_{i}^{T} P_{i} \mathbb{B}_{i}+\Psi_{11} \mathbb{B}_{i}^{T} R_{i} \mathbb{B}_{i}-\gamma_{i}^{2} I & \mathbb{D}_{i}^{T} \\
\mathbb{D}_{i} & -I
\end{array}\right] \prec 0,
\end{aligned}
$$

hold, then the closed-loop system satisfies the required finite frequency specifications (15).

Proof: The LMI (17) can be obtained by employing the same procedure as proposed in [5] and due to the limit of the space, the proof is omitted.

Additionaly, it is important to stress that (17) can be adopted for the case when design specifications must hold for the entire frequency range. Only in the case when $\Theta_{i}=[0, \pi]$ in (15), one can take $\Psi=0$ in (1) and then making the similar steps to these for finite frequency rages it is possible to obtain the following result.

Theorem 2: For the state-space realization of $G_{i}$ in the form (14) if there exist $P_{i}$ such that the following LMIs

$$
\left[\begin{array}{ccc}
\mathbb{A}_{i}^{T} P_{i} \mathbb{A}_{i} & \mathbb{A}_{i}^{T} P_{i} \mathbb{B}_{i} & \mathbb{C}_{i}^{T} \\
\mathbb{B}_{i}^{T} P_{i} \mathbb{A}_{i} & \mathbb{B}_{i}^{T} P_{i} \mathbb{B}_{i}-\gamma_{i} I & \mathbb{D}_{i}^{T} \\
\mathbb{C}_{i} & \mathbb{D}_{i} & -I
\end{array}\right] \prec 0,
$$

hold, then the closed-loop system satisfies the required entire frequency specifications.

Proof: The proof can be determined as an obvious consequence of Theorem 1 when $\Psi=0$ and thus omitted here.
With the computed $H(z):\left(A_{h}, B_{h}, C_{h}, D_{h}\right)$ the designed feedback controller matrices in (6) are then given by

$$
\begin{aligned}
& A_{c}=\left[\begin{array}{cc}
A+B D_{c} C-B K-L C-B D_{h} C & B C_{h} \\
-B_{h} C & A_{h}
\end{array}\right], \\
& B_{c}=\left[\begin{array}{c}
L+B D_{h} \\
B_{h}
\end{array}\right], C_{c}=\left[\begin{array}{ll}
-K+D_{h} C & C_{h}
\end{array}\right], D_{c}=D_{h} .
\end{aligned}
$$

\section{DESIGN OF A LEARNING CONTROLLER}

Based on the results obtained in the previous section, i.e. by considering that there exist stabilizing feedback controller $C(z)$ satisfying multi-objective performance requirements and hence the sensitivity function $S_{P}(z)$ of (3) has been determined, one can develop an effective procedure for designing the learning controller $L(z)$. The goal is to compute matrices $A_{L}, B_{L}, C_{L}$ and $D_{L}$ that define its minimal statespace realization of $L(z)$. Furthermore, since the bandwidth of the reference signal affects the convergence ratio the most, it is relevant to impose performance specifications over this specific frequency range. To proceed, it is routine to show that (4) limited to such a frequency range is equivalent to

$$
\left[\begin{array}{c}
M\left(e^{j \theta}\right) \\
I
\end{array}\right]^{T}\left[\begin{array}{cc}
I & 0 \\
0 & -\gamma^{2} I
\end{array}\right]\left[\begin{array}{c}
M\left(e^{j \theta}\right) \\
I
\end{array}\right] \prec 0, \forall \theta \in \Theta,
$$

Moreover choosing $\Pi=\operatorname{diag}\left\{I,-\gamma^{2} I\right\}$, where $\gamma$ satisfyies $0<\gamma \leq 1$, and using Lemma 1 makes (20) equivalent to (1). Interested reader is referred to [6] for more details on the finite frequency rages for ILC design. The crucial problem here is that (1) is not convex due to the product of the $L$-filter parameters and the matrices $P$ and $R$.

In order to obtain a convex formulation of the problem, again we assume that $L(z)$ has the form of $n$-th order FIR filter given by

$$
L(z)=\alpha_{0}+\alpha_{1} z^{-1}+\alpha_{2} z^{-2}+\ldots+\alpha_{n} z^{-n},
$$

and the associated minimal state-space realization is

$$
L(z)=C_{L}\left(z I-A_{L}\right)^{-1} B_{L}+D_{L},
$$

with matrices

$$
\begin{aligned}
A_{L} & =\left[\begin{array}{cc}
0 & I_{n-1} \\
0 & 0
\end{array}\right], B_{L}=\left[\begin{array}{l}
0 \\
1
\end{array}\right], \\
C_{L} & =\left[\begin{array}{lllll}
\alpha_{n} & \alpha_{n-1} & \cdots & \alpha_{2} & \alpha_{1}
\end{array}\right], D_{L}=\left[\alpha_{0}\right] .
\end{aligned}
$$

In the above form, the parameters $\alpha_{0}, \alpha_{1}, \ldots, \alpha_{n}$ to be designed are only present in the matrices $C_{L}$ and $D_{L}$. This agian allows to avoid calculation of products of two matrix variables in their entries and hence the filter parameters can be computed via a constrained convex optimization procedure using LMIs. To see this, let introduce matrices $A_{s p}, B_{s p}, C_{s p}$ and $D_{s p}$ of the state-space realization of the sensitivity function $S_{P}(z)$. Then a state-space representation of $L(z) S_{P}(z)$ in (3) is

$$
\begin{aligned}
\mathcal{A} & =\left[\begin{array}{cc}
A_{s p} & 0 \\
B_{L} C_{s p} & A_{L}
\end{array}\right], \mathcal{B}=\left[\begin{array}{c}
B_{s p} \\
B_{L} D_{s p}
\end{array}\right], \\
\mathcal{C} & =\left[\begin{array}{ll}
D_{L} C_{s p} & C_{L}
\end{array}\right], \mathcal{D}=D_{L} D_{s p} .
\end{aligned}
$$


It should be kept in mind that filter parameters appear in $\mathcal{C}$ and $\mathcal{D}$ only. Then the following result gives an LMI design for the $L$-filter.

Theorem 3: Let $\Psi=\left[\begin{array}{ll}\Psi_{11} & \Psi_{12} \\ \Psi_{12}^{*} & \Psi_{22}\end{array}\right]$ be given. Then a stable $n$-order filter $L(z)$ of the form (21) can be designed such that the ILC convergence condition (20) holds for a chosen finite frequency range $\Theta$ of Lemma 1 if there exist matrices $P, R \succ 0, \mathcal{C}$, and $\mathcal{D}$ such that the following LMI is feasible

$$
\begin{aligned}
& {\left[\begin{array}{c}
-\mathcal{A}^{T} P \mathcal{A}+\Xi_{11} \mathcal{A}^{T} R \mathcal{A}+\Xi_{12} \mathcal{A}^{T} R+\Xi_{12}^{T} R \mathcal{A}+P+\Xi_{22} R \\
-\mathcal{B}^{T} P \mathcal{A}+\Xi_{11} \mathcal{B}^{T} R \mathcal{A}+\Xi_{12} \mathcal{B}^{T} R \\
-\mathcal{C}
\end{array}\right.} \\
& \left.\begin{array}{cc}
-\mathcal{A}^{T} P \mathcal{B}+\Xi_{11} \mathcal{A}^{T} R \mathcal{B}+\Xi_{12}^{T} R \mathcal{B} & -\mathcal{C}^{T} \\
-\mathcal{B}^{T} P \mathcal{B}+\Xi_{11} \mathcal{B}^{T} R \mathcal{B}-\gamma^{2} I & 1-\mathcal{D}^{T} \\
1-\mathcal{D} & -1
\end{array}\right] \prec 0 .
\end{aligned}
$$

Moreover, the required $Q(z)$-filter can be selected as a lowpass filter with the cut-off frequency equal to the highest frequency for which the above result is valid.

Proof: This is given for the low frequency (LF) range since this choice is often encountered in physical applications and the others follow by identical steps. For the considered frequency range, the matrix $\Psi$ in (1) can be partitioned as

$$
\Psi=\left[\begin{array}{l|l}
\Psi_{11} & \Psi_{12} \\
\hline \Psi_{12}^{T} & \Psi_{22}
\end{array}\right]=\left[\begin{array}{l|c}
0 & 1 \\
\hline 1 & -2 \cos \left(\varpi_{l}\right)
\end{array}\right]
$$

and with the notation introduced in (24), the state-space representation of $M(z)$ in (3) for $Q(z)=1$ is

$$
\left[\begin{array}{c|c}
\mathcal{A} & \mathcal{B} \\
\hline-\mathcal{C} & 1-\mathcal{D}
\end{array}\right]
$$

Direct application of Lemma 1 for the above state-space model matrices gives (25) and the proof is complete.

Remark 1: The robustness filter $Q(z)$ can be implemented as a zero-phase filter (e.g. by using the filtfilt routine in MATLAB) since such filtering is performed off-line using previous trial information and the known reference trajectory.

Remark 2: To minimize the inaccuracies between the computed $L$ and the known $S_{P}^{-1}$, the term $\gamma$ in (25) has to be minimized. This can be achieved by using the linear objective minimization procedure

$$
\min _{R \succ 0, \mu>0} \mu
$$

subject to $(25)$

substituting $\mu=\gamma^{2}$.

\section{A COMPLETE DESIGN PROCEDURE}

To summarize the paper developments, a design procedure for ILC scheme is given as follows.

Step 1: Compute the matrices $K$ and $L$ using LQG control approach, e.g. using MATLAB routines dlqr() and dlqe () .

Step 2: Impose the performance specification in finite and/or entire frequency range based on required shape of sensitivity function.
Step 3: Solve the LMIs (17)-(18) and compute the feedback controller parameters from (19).

Step 4: Check the frequency content of the reference signal and carry out the finite frequency range design of the learning filter with Theorem 3.

Step 5: Obtain the learning controller parameters from (23).

\section{DESIGN EXAMPLE WITH AN EXPERIMENTAL VALIDATION}

To validate the proposed controller design procedures, the results of an experimental validation on a laboratory servomechanism system are presented and discussed. The system consists of a DC motor and the inertial mass (brass cylinder, weight $2.03[\mathrm{~kg}]$, diameter $0.066[\mathrm{~m}]$, length 0.068 $[\mathrm{m}]$ ), which are connected through a rigid shaft. Rotational motion of the mass is exited by the DC motor, load position is measured by an incremental encoder and the whole system operates with a PC-based digital controller. The system is fully integrated with MATLAB/SIMULINK and operates in real-time. The block scheme of the servo with the direct connection to the representation (5) is shown in Fig. 2 where $J=11.18 \cdot 10^{-4}\left[\mathrm{~kg} \cdot \mathrm{m}^{2}\right]$ is the moment of inertia, $B=3.5077 \cdot 10^{-6}[\mathrm{~N} \cdot \mathrm{m} \cdot \mathrm{s}]$ is viscous friction coefficient, $K_{e}=0.1288\left[\frac{V}{\mathrm{rad} / \mathrm{s}}\right]$ is the electromotive force constant, $K_{m}=0.0163\left[\frac{N \cdot m}{A}\right]$ is the motor torque constant, $R=2[\Omega]$ is the resistance and $L=0.001[H]$ stands for the inductance. A continuous-time model (see the block diagram of the

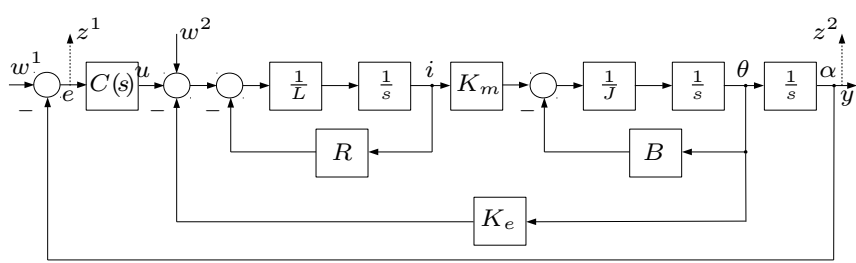

Fig. 2. Block scheme of the modular servo

system depicted in Fig. 2) has been transformed to the discrete state-space form using the sampling time $T_{s}=0.01$ and the resulting matrices of the state-space model are

$$
\begin{aligned}
& A=\left[\begin{array}{ccc}
-0.0005 & -0.0639 & 0 \\
0.0072 & 0.9911 & 0 \\
0.0001 & 0.01 & 1
\end{array}\right], B=\left[\begin{array}{l}
0.4958 \\
0.0690 \\
0.0003
\end{array}\right], \\
& C=\left[\begin{array}{lll}
0 & 0 & 24
\end{array}\right], D=0 .
\end{aligned}
$$

Moreover, according to the representation (5) we have $B_{x_{1}}=$ $0, B_{x_{2}}=B, C_{z_{1}}=-C, C_{z_{2}}=C, D_{z_{1}}=1, D_{1}=0$, $D_{z_{2}}=0, D_{2}=0, D_{y_{1}}=0$ and $D_{y_{2}}=0$. it means that $z^{1}=e, z^{2}=y$, and $y=\alpha$. The state vector $x=[i \omega \alpha]^{T}$. The reference trajectory for the mass position is of duration 6.5 secs as shown in Fig. 3 and consists of harmonic components from 0 to $2[\mathrm{~Hz}]$. This means that the frequency range from 0 to $2[\mathrm{~Hz}]$ is of primary importance. The reference signal consists of 6 rotations in the positive direction, a return path, 6 rotations in the negative direction and then a return to the start position. Based on the separation rule and using the simple pole placement technique the 


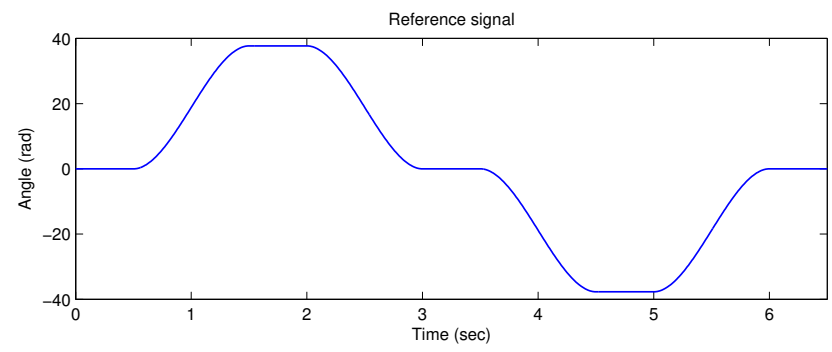

Fig. 3. The reference trajectory

observer gain $L=\left[\begin{array}{lll}0.0039 & -0.0598 & -0.0074\end{array}\right]^{T}$ and the gain matrix $K=[-0.0170-2.3440-21.9580]$ are derived. Furthermore, assuming that the initial controller (6) is a static one with $D_{c}=0.0001$, and solving the set of LMIs (17) yields the feasible solution and the application of the feedback controller design procedure gives the corresponding stabilizing controller matrices as

$$
\begin{aligned}
& A_{c}=\left[\begin{array}{rrr}
-0.0089 & -1.2260 & -10.7572 \\
0.0061 & 0.8294 & -2.9437 \\
0.0001 & 0.0092 & 0.8155
\end{array}\right], B_{c}=\left[\begin{array}{r}
0.0054 \\
-0.0595 \\
-0.0074
\end{array}\right] \text {, } \\
& C_{c}=[-0.0170-2.3440-21.8811], D_{c}=[0.0032] \text {. }
\end{aligned}
$$

With the above data, the design procedure for the learning controller for frequency range from 0 to $2[\mathrm{~Hz}]$ has been executed and gives the following FIR polynomial coefficients

$$
\alpha_{0}=7.7126, \alpha_{1}=\left[\begin{array}{ll}
3.9546 & -11.1428
\end{array}\right] \text {. }
$$

Given (23) and (26) a state-space model of the learning controller is given by the matrices

$$
A_{L}=\left[\begin{array}{ll}
0 & 1 \\
0 & 0
\end{array}\right], B_{L}=\left[\begin{array}{l}
0 \\
1
\end{array}\right], C_{L}=[3.955-11.14], D_{L}=7.713
$$

Figure 4 shows that the frequency responses of $L(z)$ and $S_{P}(z)$ are almost identical in the low frequency range up to $10[\mathrm{~Hz}]$. Moreover, the test system has a tendency to amplify high frequency signals (noise) and hence $Q(z)$ should be chosen as a low-pass filter with the cut-off frequency below $10[\mathrm{~Hz}]$. In this example a sixth order low-pass digital Butterworth filter with cut-off frequency equal to $4[\mathrm{~Hz}]$ has been used. With the design completed, the resulting ILC scheme was experimentally tested over 20 trials. After each trial the

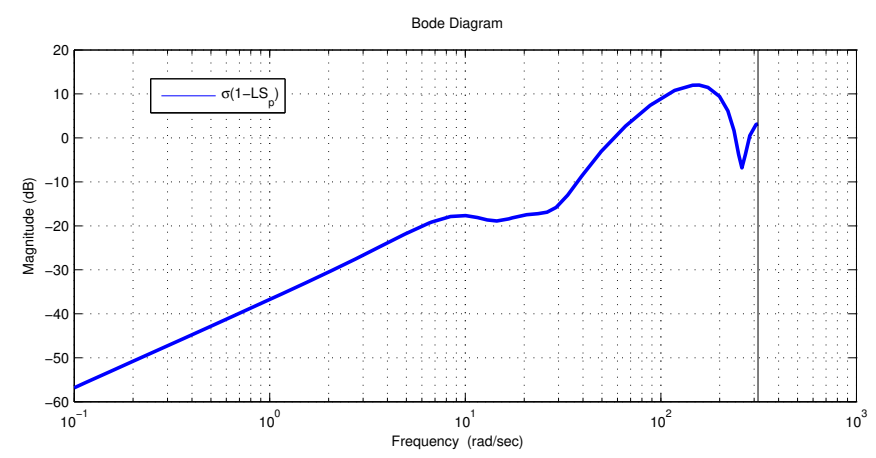

Fig. 4. Magnitude plot of $\left(1-L S_{P}^{-1}\right)$.

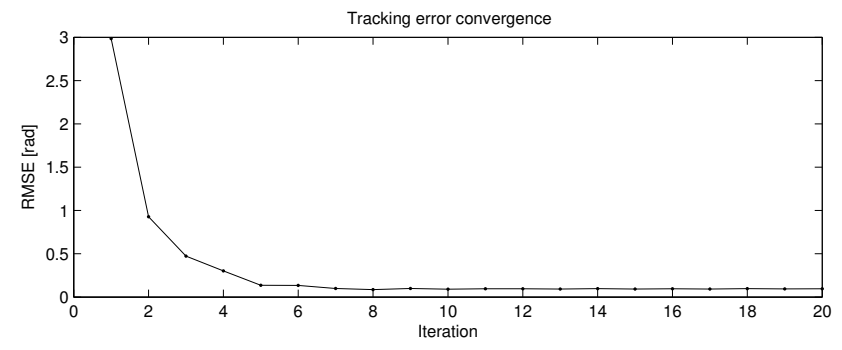

Fig. 5. Convergence of the tracking error

corresponding root mean square (RMS) value of the tracking error was computed using

$$
\mathrm{RMS}=\sqrt{\frac{1}{N} \sum_{p=1}^{N} e(p)^{2}}
$$

where $N$ is the number of sampled data in the trial time. In Fig. 5 the RMS value of the tracking error is shown as a function of the trial number. Clearly, the last figure confirms convergence of the tracking error with increasing $k$.

\section{CONCLUSIONS}

In this paper a systematic procedure for the design of both feedback and learning controllers for ILC schemes has been developed. Using the proposed design it is possible to impose many control performance constrains over finite and/or semi-finite frequency ranges. It is shown that FIR form of required controllers leads to a problem form in terms of LMIs. Experimental validation of the results on a laboratory setup has also been presented demonstrating the validity of the proposed design.

The area for possible future research is to use the twodimensional/repetitive systems setting. Application of these systems setting should result in one step design procedure and lead to an optimal complementation of the feedback with the learning action. This means that interplay between feedback and feedforward actions has to be considered. Also robust control should be investigated.

\section{REFERENCES}

[1] H.-S. Ahn, Y.-Q. Chen, and K. L. Moore, "Iterative learning control: brief survey and categorization," IEEE Transactions on Systems, Man and Cybernetics, Part C, vol. 37, no. 6, pp. 1109-1121, 2007.

[2] D. A. Bristow, M. Tharayil, and A. Alleyne, "A survey of iterative learning control," IEEE Control Systems Magazine, vol. 26, no. 3, pp. 96-114, 2006.

[3] T. Iwasaki and S. Hara, "Generalized KYP lemma: unified frequency domain inequalities with design applications," IEEE Transactions on Automatic Control, vol. 50, no. 1, pp. 41-59, 2005.

[4] S. Boyd and C. Barratt, Linear Controller Design: Limits of Performance, ser. Information and System Sciences. Prentice-Hall, 1991.

[5] X. Zhang and G. Yang, "Multi-objective control synthesis with mixed frequency small gain specifications: Youla parametrization approach," in Proceedings of the Chineese Control and Decision Conference, CCDC 2008, 2008, pp. 5267-5272.

[6] W. Paszke, E. Rogers, and K. Gałkowski, "Experimentally verified generalized KYP lemma based iterative learning control design," Control Engineering Practice, vol. 53, no. 10, pp. 57-67, 2016. 